\title{
PROMONTOFIJ ACIÓN VERSUS IVS POSTERIOR EN EL TRATAMIENTO DE LOS PROLAPSOS APICALES
}

\author{
Carlos Rondini F-D. ${ }^{1}$, Fernando Troncoso R. ${ }^{1}$, Boris Moran V. ${ }^{1}$, Gonzalo Vesperinas A. ${ }^{1}$, \\ Marcos Levancini A. ${ }^{1}$, Carlos Troncoso R. ${ }^{1}$ \\ ${ }^{1}$ Unidad de Ginecología, Hospital Padre Hurtado, Servicio de Salud Metropolitano Sur Oriente.
}

\begin{abstract}
RESUMEN
Objetivo: comparar aspectos del intra y postoperatorio inmediato entre dos técnicas quirúrgicas para el tratamiento de prolapsos apicales. Material y métodos: Estudio retrospectivo, entre marzo de 2000 y agosto de 2004, de 50 pacientes con prolapso apical, de cúpula vaginal o procidencia uterina que fueron corregidas quirúrgicamente mediante sacropromontofijación (SPF) o IVS posterior (posterior intravagynalslingplasty) con o sin histerectomía según correspondiera. Se compararon tiempos operatorios, complicaciones intra y post operatorias y estadía hospitalaria. Resultados: Los grupos fueron comparables, con excepción del antecedente de fórceps que fue significativamente mayor en el grupo de pacientes sometidas a IVS posterior ( 0 vs $0,1 \pm 0.4, p=0,04)$. El tiempo operatorio promedio, independiente si hubo histerectomía asociada, fue significativamente mayor para el grupo SPF $(60,5 \pm 29$ minutos vs $86,6 \pm 22$ minutos; $p=0,01)$. La estadía postoperatoria promedio fue significativamente menor en el grupo IVS posterior $(2,0 \pm 0,6 \mathrm{vs}$ $3,2 \pm 0,6$ días; $p<0,01)$. Hubo una tendencia no significativa de una menor frecuencia de complicaciones intra $(4,2 \%$ vs $7,7 \% ; p=0,6)$ y post operatorias inmediatas $(8 \%$ vs $26 \% ; p=0,08)$ en el grupo IVS posterior. Conclusión: Nuestro estudio sugiere que en relación a tiempo operatorio y estadía hospitalaria el IVS posterior con o sin histerectomía asociada ofrece claros beneficios sobre la técnica de SPF, con una tasa similar de complicaciones intra y postoperatorias.
\end{abstract}

\section{PALABRAS CLAVES: Prolapso apical, IVS posterior, colposacropexia}

\section{SUMMARY}

Objective: Compare intra and immediate postoperative aspects between two surgical techniques for apical prolapse treatment. Study design: A retrospective study was performed. Between March 2000 and August 2004, 50 patients with vault or uterine prolapse were treated surgically by colposacropexia (SPF) or posterior intravaginal sling plasty (PIVS). If hysterocele was present, a hysterectomy was performed prior to the procedure. Surgical time, intra and immediate post operative complications and hospital stay were the main end points evaluated. Results: The epidemiologic profile was similar in both groups. The only exception was forceps delivery, which was significantly more frequent in PIVS group ( 0 vs $0.1 \pm 0.4 ; p=0.04)$. Surgical time with or without hysterectomy was significantly higher in SPF group ( $86.6 \pm 22 \min v s 60.5 \pm 29 \mathrm{~min}$; $\mathrm{p}=0.01)$. Mean hospital stay was significantly lower in IVSP groups $(2.0 \pm 0.6$ vs $3.2 \pm 0.6$ days; $p<0.01)$. PIVS showed not significant lower tendency to intra (4.2\% vs $7.7 \% ; p=0.6)$ and post operative $(8 \%$ vs $26 \%$; $\mathrm{p}=0.08$ ) complications. Conclusion: PIVS offers clear advantages over SPF in apical prolapse treatment when surgical time and hospital stay are compared, with similar intra and post operative complications.

KEY WORDS: Apical prolapse, posterior IVS, colposacropexia 


\section{INTRODUCCIÓN}

En 1992 Delancey divide el tejido conectivo que sirve de sostén a la vagina en tres niveles: Nivel I o apical; Nivel II o medio vaginal y Nivel III o distal (1). En el primer nivel el tercio superior de la vagina es suspendida al sacro y la pared pelviana lateral mediante el complejo de ligamentos útero sacro-cardinal. Los defectos a este nivel dan origen a los prolapsos apicales los que incluyen: histeroceles, enteroceles y prolapsos de cúpula vaginal (1).

La promontofijacion vía abdominal es considerada el "estándar dorado" de las cirugías para la corrección de defectos apicales dado que cuenta con seguimientos de hasta 17 años con tasas de curación cercanas al $90 \%(2,3,4)$. Sin embargo, es una cirugía que implica destrezas quirúrgicas que incluyen manejo en el retroperitoneo con una tasa de complicaciones intraoperatorias (hemorragia, hematoma retroperitoneal, lesión ureteral) y post operatorias (incontinencia urinaria de novo, erosión de la malla, fístulas a vejiga y recto, osteomielitis sacra) no despreciables $(5,6)$. Esto ha impulsado el desarrollo de nuevas técnicas más sencillas y con menores complicaciones. Es así como en 1997 Petros describe la técnica de IVSP (intravaginal slingplasty posterior), la cual esta basada en la técnica de TVT (tensión free vaginal tape) y pretende formar un neoligamento que suspenda la cúpula vaginal y reemplace el complejo útero sacrocardinal dañado (7).

Para evaluar los resultados de la introducción de esta nueva técnica comparamos los primeras 24 cirugías de IVSP realizados en el servicio con 26 pacientes sometidas a sacropromonto fijación (SPF) por vía abdominal

\section{MATERIAL Y MÉTODOS}

Evaluamos retrospectivamente los procedimientos quirúrgicos de la Unidad de Ginecología del Hospital Padre Hurtado, realizados entre marzo de 2000 y agosto de 2004, de 50 pacientes sometidas a corrección de prolapso de cúpula vaginal o procidencia genital ya sea mediante SPF o IVSP.

Las primeras fueron realizadas desde el inicio del funcionamiento de la Unidad en marzo de 2000 hasta diciembre de 2003. Los IVSP fueron realizados durante el primer semestre de 2004. Todas las pacientes fueron evaluadas y caracterizadas según la clasificación POP Q (8). Todas las pacientes que deseaban mantener actividad sexual y que su punto $D$ (fondo de saco vaginal posterior) se encontraba $1 \mathrm{~cm}$ distal al himen (grado III) fueron candidatas a una de las dos técnicas.

En aquellas pacientes que presentaban histerocele se realizo inicialmente histerectomía abdominal o vaginal según la vía de abordaje.

Para las SPF en el servicio utilizamos la técnica clásica, que brevemente pretende interponer un puente de material sintético (malla de polipropileno) entre el promontorio sacro y la cúpula vaginal (9). La técnica de IVSP se realizó mediante una colpotomía transversa a nivel de la cúpula vaginal, sutura de una malla de polipropileno de $1,5 \mathrm{~cm}$ de ancho y $30 \mathrm{~cm}$ de largo de prolene \#1, disección hacia fosa isquiorectal e inserción de aguja tipo PIMET $^{\circledR}$ a $3 \mathrm{~cm}$ bajo y lateral al esfínter anal, se enhebra la aguja y se repite el movimiento inverso (10).

Se evaluó tiempo operatorio, complicaciones intraoperatorias, estadía hospitalaria y complicaciones post operatorias inmediatas, definidas como aquellas que se presentan dentro del primer mes post operatorio. Se considero falla del tratamiento la persistencia del prolapso de igual o mayor severidad dentro del primer mes post operatorio. Cuando se compararon variables categóricas se utilizó Chi cuadrado y para variables continuas ANOVA; las comparaciones múltiples fueron efectuadas según Bonferroni. Se consideró como significativo un $p<0,05$.

\section{RESULTADOS}

La edad promedio, el IMC, la paridad, el antecedente de cesáreas y de patología pulmonar no fue distinto entre los grupos; solo el antecedente de fórceps fue significativamente más frecuente en el grupo de pacientes sometidas a IVS posterior $(p=0,04)$ (Tabla I). Los tiempos operatorios

\section{Tabla I}

\section{CARACTERÍSTICAS EPIDEMIOLÓGICAS SEGÚN GRUPOS DE ESTUDIO}

\begin{tabular}{lccl}
\hline Variable & SPF $(n: 26)$ & IVS $(n: 24)$ & Valor $p$ \\
\hline Edad & $56,4 \pm 7,6$ & $60,4 \pm 12,3$ & $0,06^{*}$ \\
IMC & $28,6 \pm 4,5$ & $29,0 \pm 4,8$ & $0,9^{*}$ \\
Paridad & $3,5 \pm 1,9$ & $4,5 \pm 2,8$ & $0,17^{*}$ \\
PV & $3,4 \pm 1,9$ & $4,2 \pm 2,8$ & $0,20^{*}$ \\
Fórceps & 0 & $0,1 \pm 0,4$ & $0,04^{* *}$ \\
Cesárea & $0,19 \pm 0,5$ & $0,13 \pm 0,3$ & $0,56^{*}$ \\
PR & $11,5 \pm 0,5$ & $4,2 \pm 0,1$ & $0,33^{*}$ \\
\hline
\end{tabular}

IMC: índice de masa corporal; PV: parto vaginal; PR: patología respiratoria

*No significativo.

${ }^{* *}$ Student Test: $p<0,05$. 
Tabla II

\section{DETALLES OPERATORIOS SEGÚN GRUPOS DE ESTUDIO}

\begin{tabular}{lccc}
\hline Variable & SPF $(n: 26)$ & IVS $(n: 24)$ & Valor $p$ \\
\hline Tiempo operatorio & $86,6 \pm 22,8$ & $60,5 \pm 29,5$ & $0,01^{*}$ \\
Días hospitalización & $3,2 \pm 0,6$ & $2,0 \pm 0,6$ & $<0,01^{*}$ \\
CIO & $7,7 \%$ & $4,2 \%$ & $0,6^{* *}$ \\
CPO & $26,0 \%$ & $8,0 \%$ & $0,08^{* *}$ \\
\hline
\end{tabular}

*Student Test: $p<0,05$.

${ }^{*}$ No significativo.

CIO: Complicación intraoperatoria.

CPO: Complicación postoperatoria.

promedios independiente si se realizó o no histerectomía asociada fueron significativamente mayores para el grupo SPF $(p=0,01)$; de la misma manera hubo diferencia significativa en la estadía post operatoria promedio $(p<0,01)$ siendo menor en las pacientes sometidas a IVSP (Tabla II). Cuando se analizaron subgrupos de pacientes en los que se realizó o no histerectomía, los tiempos operatorios continuaron siendo significativamente mayores en las pacientes sometidas a SPF (Tabla III). Hubo una tendencia no significativa hacia una menor frecuencia de complicaciones tanto intra como postoperatorias inmediatas en el grupo de IVSP ( $p=0,6$ y $p=0,08$ respectivamente) (Tabla IV). La tasa de fallas fue comparable entre los grupos (Tabla V).

\section{DISCUSIÓN}

Múltiples técnicas para suspender la cúpula vaginal en pacientes histerectomizadas 0 en el tiempo de la histerectomía han sido descritas. Sin embargo, existen escasos trabajos prospectivos randomizados que las hayan comparado. Además existen muchas modificaciones a las técnicas originales lo cual complica aun más la comparación

Tabla III

\section{TIEMPO OPERATORIO (MINUTOS) SEGÚN SUBGRUPOS DE ESTUDIO}

\begin{tabular}{lccccc}
\hline Variable & $\begin{array}{c}S P F \\
(n \text { 15) }\end{array}$ & $\begin{array}{c}S P F+H T \\
(n: 11)\end{array}$ & $\begin{array}{c}\text { IVS } \\
(n: 11)\end{array}$ & $\begin{array}{c}\text { IVS }+H V \\
(n \text { 13) }\end{array}$ & $\begin{array}{c}\text { Valor } \\
p\end{array}$ \\
\hline $\begin{array}{l}\text { Tiempo } \\
\text { operatorio }\end{array}$ & $75 \pm 16$ & $101 \pm 21$ & $44 \pm 24$ & $74 \pm 27$ & $<0,01$ \\
\hline
\end{tabular}

Test de Bonferroni: SPF vs IVS, $\mathrm{p}<0,008$; SPF+HT vs IVS+HV, $p<0,027$
Tabla IV

\section{COMPLICACIONES INTRAOPERATORIAS Y POSTOPERATORIAS}

\begin{tabular}{lr}
\hline Complicación & $n$ \\
\hline SPF & 2 \\
Hemorragia & 6 \\
Infección & 1 \\
Osteocondritis & \\
IVS & 1 \\
Lesión vesical & 2 \\
Infección & \\
\hline
\end{tabular}

entre estas. No obstante la sacropromontofijacion continua siendo la técnica de elección en pacientes con prolapsos apicales dado sus altas tasas de curación, mayores al 90\%, con seguimientos incluso hasta 17 años. No obstante es una técnica que requiere tiempo, destreza quirúrgica y no esta exenta de complicaciones incluso graves a saber: hematomas retroperitoneales, lesión ureteral, osteomielitis sacra y otras.

En 1997, Petros describe la técnica de IVS posterior (8) y más tarde en 2002 Farnsworth (11) publica el primer trabajo prospectivo. Se trata de una técnica mínimamente invasiva basada en la técnica de TVT (tensión free vaginal tape) y que pretende superar las dificultades de la sacropromontofijacion y dar solución vía vaginal al prolapso.

En nuestro trabajo pudimos comprobar que esta técnica permite acortar tanto los tiempos operatorios ya sea cuando de realiza como cirugía única o asociada a histerectomía. De la misma manera las estadías post operatorias se acortaron de manera significativa. En relación a las complicaciones intraoperatorias estas se refieren a 2 complicaciones hemorragíparas en pacientes sometidas a SPF y una lesión vesical en el grupo de

TABLA V

SEGUIMIENTO A CORTO PLAZO SEGÚN GRUPOS DE ESTUDIO*

\begin{tabular}{lccc}
\hline Variable & $S P F(n: 26)$ & IVS $(n: 24)$ & Valor $p$ \\
\hline Con mejoría & 25 & 24 & NS \\
Sin mejoría & 1 & 1 & NS \\
\hline
\end{tabular}

* Seguimiento de mejoría a 1 mes respecto evaluación al momento del alta.

NS: no significativo. 
IVSP. En ambos grupos estas se produjeron en el tiempo de la histerectomía y no son directamente atribuibles a la técnica de corrección de prolapso apical.

En relación a las complicaciones post operatorias esta se refiere fundamentalmente a complicaciones infecciosas en relación a la cicatriz de laparotomía y celulitis de cúpula vaginal en el grupo de las SPF. Solo una complicación seria se registro en el grupo de SPF y se trato de una paciente que evolucionó con una osteomielitis sacra la cual requirió tratamiento antibiótico prolongado y posterior retiro de la malla por laparotomía. El cuadro fue confirmado por histología.

En el grupo de IVS posterior se registró una celulitis de la colporrafia que se trató con antibióticos ambulatorios. Si bien existió una clara menor tendencia de complicaciones postoperatorias en el grupo de IVSP esta no alcanzo significación estadística debido probablemente al número reducido de pacientes en ambas grupos y a la baja frecuencia de ocurrencia. Los resultados a corto plazo no demostraron diferencias significativas.

En nuestro medio Silva y cols en 2004 informan por primera vez una serie de 16 casos con esta técnica, el seguimiento promedio a 70 días no mostró complicaciones, ni recidivas; el tiempo promedio solo para realizar IVSP fue de 23 minutos con una pérdida sanguínea promedio de 100 $\mathrm{ml}(12)$.

Finalmente esta por resolverse si el seguimiento a largo plazo de estas pacientes demuestra tasas de curación comparable o superior a la técnica clásica de SPF.

\section{BIBLIOGRAFÍA}

1. Delancey JO. Anatomic aspects of vaginal eversion after hysterectomy. Am J Obstet Gynecol 1992; 166: 1717-1724.
2. Lefranc JP, Atallah D, Camatte S, Blondon J. Long term follow up of posthisterectomy vagynal vault prolapse abdominal repair; a report of 85 cases. J Am Coll Surg 2002; 195: 352-58.

3. Timmons MC, Addison WA, Addison SB et al. Abdominal sacral colpopexy in 163 women with posthystectomy vaginal vault prolapse and enterocele. J Reprod Med 1992; 37: 323-27.

4. Benson JT, Lucente V, McClellan E. Vaginal versus abdominal reconstructive surgery for the treatment of pelvic support defects. A prospective randomized study with long term outcome evaluation. Am J Obstet Gynecol 1996: 175; 1418-22.

5. Culligan PJ, Murphy M, Blackwell L, Hammons G, Graham C, Heit MH. Long term success of abdominal sacral colpopexy using synthetic mesh. Am J Obstet Gynecol 2002; 187: 1473-80.

6. Kohli N, Walsh PM, Roat TW, Karraman MM. Mesh erosion after abdominal sacral colpopexy. Obstet Gynecol 1998; 92: 999-1004.

7. Visco AG, Weidner AC, Barner MD et al. Vaginal mesh erosion after abdominal sacro colpopexy. Am J Obstet Gynecol 2001; 184: 297-302.

8. Petros PE. New ambulatory surgical methods using an anatomical classification of urinary dysfunction improve stress, urge and abnormal emptying. Int Urogynecol J Pelvic Floor Disfunct 1997; 8: 270-7.

9. Bump RC, Mattiasson A, Bo K, Brubaker LP , John A, DeLancey JA et al. The standardization of terminology of pelvic organ prolapse and pelvic floor dysfunction. Am J Obstet Gynecol 1996; 175: 10-7.

10. Cundiff GW, Addison A. Management of pelvic organ prolapse. Obstetrics Gynaecology Clin North Am 1998; 4(25): 907-21.

11. Farnsworth BN. Posterior intravaginal slingplasty (infracoccygeal sacropexy) for severe post hysterectomy vaginal vault prolapse-a preliminary report on efficacy and safety. Int Urogynecol J Pelvic Floor Dysfunct; 2002; 13(1): 4-8.

12. Silva A, Ferrada L, Arroyo C, Nalbandian K. IVS posterior (posterior intravaginal slingplasty) para el tratamiento de prolapso de cúpula vaginal o procidencia de útero: serie de casos. ReV CHIL OBstet GINECOL 2004; 69(6): 419-24. 